BULL. AUSTRAL. MATH. SOC.

VOL. $21(1980), 7-12$.

\title{
FURTHER INEQUALITIES FOR CONVEX SETS WITH LATTICE POINT CONSTRAINTS IN THE PLANE
}

\author{
P.R. SCOTT
}

Let $K$ be a bounded closed convex set in the plane containing no points of the integral lattice in its interior and having width $w$, area $A$, perimeter $p$ and circumradius $R$. The following best possible inequalities are established:

$$
\begin{aligned}
& (w-1) A \leq \frac{3}{2} w^{2}, \\
& (w-1) p \leq 3 w, \\
& (w-1) R \leq w / \sqrt{3} .
\end{aligned}
$$

\section{Introduction}

Let $K$ be a bounded, closed, convex set in the euclidean plane, containing no points of the integral lattice in its interior. We denote the diameter, width, perimeter, area, inradius and circumradius of $K$ by $d, w, p, A, r$ and $R$ respectively.

It is known [3] that the width satisfies

$$
w \leq \frac{1}{2}(2+\sqrt{ } 3)
$$

with equality when and only when $K$ is an equilateral triangle $E$, of side length $(2+\sqrt{3}) / \sqrt{3}$. It has also been recently established [5] that

$$
(w-1)(d-1) \leq 1 \text {, }
$$

or equivalently,

$$
(w-1) d \leq w
$$

Received 19 June 1979. 
with equality when and only when $K$ is a triangle of diameter $d$ and width $w=d /(d-1)$ (Figure 1).

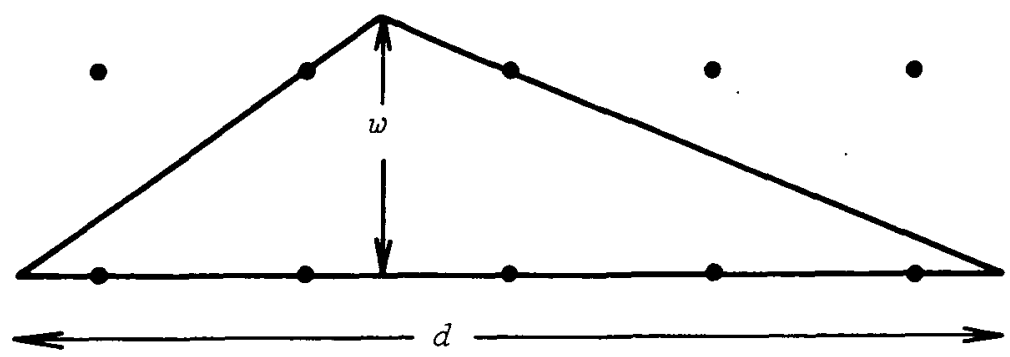

Figure 1

We shall prove several analogous results.

THEOREM 1. $(w-1) A \leq \frac{1}{2} w^{2}$, with equality when and only when $K$ is a triangle of width $w$ and diameter $w /(w-1)$ (Figure 1 ).

THEOREM 2. $(w-1) p \leq 3 w$ with equality when and only when $K=E$.

THEOREM 3. $(w-1) R \leq w / \sqrt{ } 3$ with equality when and only when $K=E$.

According to Blaschke's Theorem [1], every bounded convex figure of width $w$ contains a circle of radius $w / 3$. It follows that $w \leq 3 r$; equality holds here when and only when the figure is an equilateral triangle. Using this result and (1), we obtain the following corollaries.

COROLLARIES.

$$
\begin{aligned}
& (w-1) A \leq 3 \omega r / 2 \leq 9 r^{2} / 2 ; \\
& (w-1) A \leq(7+4 \sqrt{ } 3) / 8(\approx 1.74) ; \\
& (w-1) p \leq 9 r ; \\
& (w-1) p \leq(6+3 \sqrt{ } 3) / 2 ; \\
& (w-1) R \leq \sqrt{3} \cdot r ; \\
& (w-1) R \leq(3+2 \sqrt{ } 3) / 6 .
\end{aligned}
$$

In each case we have equality when and only when $K=E$. 


\section{Proof of Theorems 2 and 3}

To establish Theorem 3, we recall a theorem of Jung [2] which states that any set of diameter $d$ is contained in a circular disc of radius $R \leq d / \sqrt{ } 3$. Theorem 3 now follows immediately from (2), since

$$
(w-1) R \leq(w-1) d / \sqrt{ } 3 \leq w / \sqrt{3} \text {. }
$$

For equality in Jung's result we require $K$ to be an equilaterial triangle; for equality in (2), $K$ must be as in Figure 1. Hence equality occurs in Theorem 3 when and only when $K=E$.

We now show that Theorem 2 can be deduced from Theorem 1 . If $K$ is any convex polygon, we can partition $K$ into triangles by joining each vertex to the (an) in-centre of $K$. Summing the areas of these triangles easily gives for $K$ the inequality

$$
A \geq \frac{1}{2} p r \text {. }
$$

Since any convex set $K$ in the plane can be approximated as closely as we please by a convex polygon, we conclude that this inequality is valid for any convex set $K$ in the plane.

Assuming the validity of Theorem 1, we now have

$$
(w-1) p \leq 2(w-1) A / r \leq w^{2} / r \leq 3 w
$$

since $w \leq 3 r$ by Blaschke's Theorem. Hence $(w-1) p \leq 3 w$ as required. It is easily seen that equality occurs here when and only when $K=E$.

We notice that the inequality of Theorem 2 follows easily from (2) in the special case when $K$ is a triangle, for then $p \leq 3 d$, and

$$
(w-1) p \leq(w-1) 3 d \leq 3 w \text {. }
$$

\section{Some preliminary results}

We observe that the statement of Theorem 1 can be written as

$$
\frac{1}{2 A}-\frac{w-1}{w^{2}} \geq 0 \text {. }
$$

We shall assume therefore that $K$ is a set for which the left hand side of this inequality is as small as possible. Since $(w-1) / w^{2}$ is an increasing function of $w$, we choose $K$ with $A, w$ as large as possible. 
Let $D$ be a largest circular disc contained in $K$, having radius $r$. It is known [4] that for any convex set $K$,

$$
(w-2 r) A \leq w^{2} r / \sqrt{ } 3 .
$$

Hence if $r \leq \frac{1}{2}$,

$$
(w-1) A \leq(w-2 r) A \leq w^{2} r / \sqrt{ } 3 \leq w^{2} /(2 \sqrt{ } 3)<w^{2} / 2 .
$$

We may therefore assume that $K$ contains a disc $D$ of radius $r>\frac{2}{2}$.

By suitably translating $K$ we may assume that the centre of $D$ lies in the interior of the square with vertices $0(0,0), B(1,0), C(1,1)$, $D(0,1)$. Since $K$ is convex, $K$ is bounded by lines through the points $O, B, C, D$. If these lines form a convex quadrilateral $Q$, then $Q$ contains no lattice points in its interior, and we may assume that $K$ is $Q$. On the other hand, these lines may determine a triangular region $T$, as for example a degenerate quadrilateral, or when a line through $D$ separates $K$ from $C$. Such a region $T$ may contain interior lattice points; nevertheless, it will be sufficient for us to establish the theorem for $T$.

\section{Proof of Theorem 1}

First let $K$ be the convex quadrilateral $Q$. The following result is established in [3].

LEMMA. The quadrilateral $Q$ can be transformed into a kite $Q^{\prime}$ having the following properties:

(a) $w\left(Q^{\prime}\right) \geq w(Q) ;$

(b) $Q^{\prime}$ contains no lattice point in its interior;

(c) $Q^{\prime}$ has its axis along the line $x=\frac{1}{2}$,

(d) the sides of $Q^{\prime}$ pass through $O, B, C, D$ respectively;

(e) $A\left(Q^{\prime}\right) \geq A(Q)$.

Property (e) is not stated explicitly in [3], but follows from the fact that $Q^{\prime}$ is obtained from $Q$ by Steiner symmetrization and enlargement with scale factor $s \geq 1$.

Clearly we may take $K$ to be the kite $Q^{\prime}=X Y Z W$ (Figure 2). Let 
$X Z=t, \quad Y W=u$. Then $2 A=t u$.

Also, computing the areas of the component parts of $Q^{\prime}$ gives

$$
\begin{aligned}
2 A & =2+(t-1) \cdot 1+(u-1) \cdot 1 \\
& =t+u .
\end{aligned}
$$

Hence

$$
t u=t+u \text {. }
$$

Suppose that $0<t \leq u$; then

$t \leq 2$. Now

$$
A=\frac{1}{2} t u=\frac{1}{2} t^{2} /(t-1)<\frac{3}{2} w^{2} /(w-1),
$$

since $w<t$, and $t^{2} /(t-1)$ is a

decreasing function of $t$ for

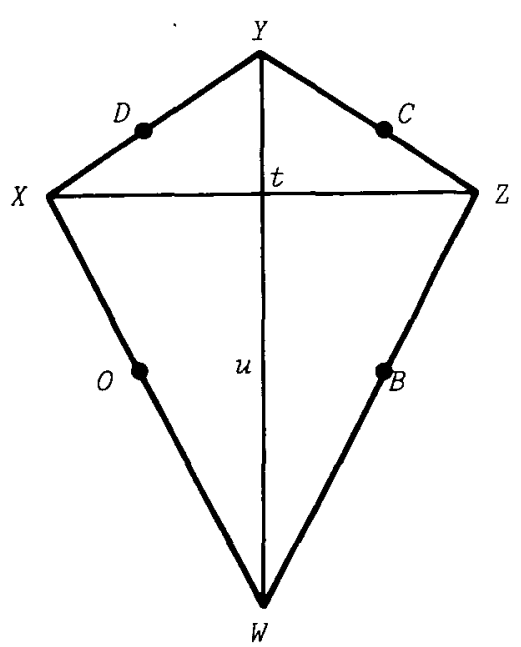

Figure 2

$0<t \leq 2$. A similar argument holds

for $0<u \leq t$.

Hence if $K$ is the quadrilateral $Q$,

$$
A(w-1)<3 w^{2}
$$

Now let $K$ be the triangle $T$. In this case

$$
A=\frac{1}{2} d \omega \leq \frac{1}{2} w^{2} /(w-1)
$$

using (2).

Thus for any $K$,

$$
(w-I) A \leq \frac{1}{2} w^{2} \text {. }
$$

Equality occurs here when and only when $K$ is a triangle as in Figure 1 .

\section{References}

[1] Wilhelm Blaschke, Kreis und Kugel (Walter de Gruyter, Berlin, 1956).

[2] Heinrich Jung, "Ueber die kleinste Kugel, die eine raumliche Figur einschliesst", J. Reine Angew. Math. 123 (1901), 241-257. 
[3] P.R. Scott, "A lattice problem in the plane", Mathematika 20 (1973), $247-252$.

[4] P.R. Scott, "A family of inequalities for convex sets", Bull. Austral. Math. Soc. 20 (1979), 237-245.

[5] P.R. Scott, "Two inequalities for convex sets with lattice point constraints in the plane", Bull. London Math. Soc. (to appear).

Department of Pure Mathematics,

University of Adelaide,

Adelaide,

South Australia 5001 ,

Australia. 